\title{
Prototipo de Celula Robotica para Manufactura
}

\author{
Israel Viveros Torresa, Sidney René Toledo Martínez ${ }^{\mathbf{b}}$, Josimar Muñoz Delgadoc, \\ Rafael Zamudio Reyes ${ }^{\mathrm{d}}$. \\ Instituto Tecnologico Superior de Alvarado, campus Medellín. Escolleras Norte S/N, Col. La Trocha \\ Alvarado, Veracruz, Ver. C.P. 91700. Tel: Tel: 01 (229) 955 4173.e-mail: 162t0053@itsm.edu.mx
}

\section{Resumen}

El presente proyecto pretende estructurar el proceso de análisis, diseño, implementación y puesta en marcha de un prototipo robótico, enfocado a procesos de manufactura, utilizando diseños de autómatas aplicados a dispositivos de maquinado industrial, herramientas de mecatrónica y aplicaciones de ingeniería de sistemas, el objetivo es desarrollar una herramienta didáctica, que permita implementar, emular y desarrollar diversos procesos de manufactura, que por medio de interfaces gráficas permitan visualizar y controlar de forma intuitiva procesos industriales de manera automatizada, impulsando el aprendizaje significativo e integral que permita una aproximación a la resolución de problemas en un entorno real, además de reducir sustancialmente la curva de aprendizaje para operadores $y$ técnicos de este tipo de industria, acercando este tipo de tecnologías a micro empresas que sean susceptibles de mejorar sustancialmente la calidad en sus procesos al tener acceso a sistemas automáticos " a la medida”. Para esto se muestran las fases de implementación de un prototipo que consta de brazos robóticos, los cuales se encargan de manipular, manufacturar en ciertos procesos, seleccionar y/o desechar elementos, así como un conjunto de actuadores neumáticos que funjan como elementos de potencia para otro tipo de maquinado, asi todos los parámetros funcionales y operativos sean cargados al sistema desde una aplicación gráfica y de sensores de retroalimentación en actuadores electromecánicos. Para el proceso de operación y maquinado de los autómatas se utilizan parámetros dimensiónales atraves de modelos de aprendizaje por parte del mismo autómata. Se muestran también las etapas que se desea desarrollar en materia de, diseño y construcción del prototipo robótico, así como la implementación de las aplicaciones para la comunicación hombre máquina, que permitan la adquisición de datos y procesen la información.

Palabras clave: Robotica, mecatrónica, interfaces, procesos de manufactura automáticos, prototipos robóticos. 


\section{Introducción}

El desarrollo de técnicas en modelado CAD y CAE ha permitido una aceleración en el proceso de generación de tecnología, así también la globalización ha permitido una alta competencia por manufacturas y procesos más eficientes lo cual nos confronta con la necesidad de una constante actualización por parte de empresas e instituciones educativas en las diferentes plataformas de diseño e implementación de prácticas, métodos de ensayo, proyectos integrales entre otros. El crecimiento de la demanda y la adopción de sistemas de robótica en Norteamérica, más específicamente en México y Canadá, mostró un crecimiento en 2015 y 2016. Parte fundamental de este desarrollo competitivo se centra en dos pilares que es equipamiento y capacitación técnica, por una parte la formación de técnicos y operarios en industria es de vital importancia a fin de ejecutar correctamente la aplicación de los dispositivos de automatización lo cual muchas veces supone una prolongada curva de aprendizaje, por otra parte la implementación de esta infraestructura siempre presenta la barrera de costos de inversión altos, debido a que los equipos de proceso automatizados muchas veces son más robustos y de propósito general con respecto a las condiciones específicas de algunos sectores que se pueden beneficiar de estas tecnologías, esta condición es lo que mayormente encarece su costos; el presente proyecto busca generar tecnología en automatización de proceso de manufactura nacional que se enfoque en hacer dispositivos a la medida de las micro empresa de tal manera ampliar significativamente la integración de más sectores productivos a esta tecnología.

\section{Metodología}

La implementación del proyecto, se estructura metodológicamente de 9 etapas o fases de desarrollo que se enumeran y describen de la siguiente forma:

1. Selección de procesos de manufactura para emulación: basado en el sector productivo de impacto se evaluan los tipos de procesos a emular con el prototipo donde se considera producción y maquinado por desprendimiento de viruta en materiales no metálicos, pintado y soldadura de punto..

2. Diseño de proceso y selección de actuadores: basados en funcionalidad y sistemas de potencia se definen las caracterisiticas operativas de los eyectores finales de trabajo, se consideran elementos neumáticos que permiten un nivel de potencia versus sistemas eléctricos de bajo consumo.

3. Aplicación de herramientas CAE y CAD: aplicables al diseño de dispositivos y elementos estructurales y simulación del proceso aplicando plataforma de diseño CAD Sketchup 08 para la elaboracion de modelos estructurales asi como la manipulación de planos "open source" de los autómatas, se implementan aplicaciones de emulación cinematica como RoboDK, GeoGebra, FreeCAD, entre otros. 
4. Selección de materiales estructurales: se define la construccion y montaje de dichos elementos.

5. Seleccion de actuadores electroneumaticos y contruccion de autómatas robóticos.

6. Implementación de sistema de adquisición de datos: se estructura la operatividad de control básico basado en IDE para Atmel 328, como sistema de adqusicion de datos para establecer mecánica de programacion por grabación de trayectorias.

7. Programacion de autómatas robóticos en modalidad "training" o "seguimiento": se efectúan rutinas de prueba a fin de validar el algoritmo aplicado para la fase inicial de programacion intuitiva.

8. Implementación de interface hombre maquina(HMI): en esta fase se define la ingeniería de software relativa a la implementación de la plataforma de comunicación hombre máquina(HMI), cumpliendo con los requisitos preestablecidos de paradigma de funcionamiento, como entorno de programación gráfico de funcionalidad directa e intuitiva.

9. Ejecución de pruebas piloto y puesta a punto de prototipo.

\section{Desarrollo de Experimentos}

\subsection{Selección del proceso de manufactura.}

Se establece un modelo didáctico o instruccional que permita emular un proceso productivo de interés en el entorno local y regional, utilizando para este propósito el area metal mecánica para la generación de productos, entendiéndose como tal, todo proceso que a través de corte desbaste y desprendimiento de viruta en general, llevando a cabo la modificación dimensional y geometrica de la materia prima. Bajo este enfoque es evidente que este sector productivo se encuentra altamente polarizado en la modalidad de operación manual y uso extensivo de mano de obra con valores variados de calificación y expertis, lo que detrimenta la producción o geneneracion de producto con la transición o rotación del personal respectivo a esta áreas especificas de esta forma se busca establecer un modelo que permita vincular la automatizacion en la ejecusion sin la imperativa necesidad de un alto o mediano grado de especialización por parte del operario.

\subsection{Diseño de proceso y selección de actuadores.}

Se preestablecen parámetros dimensionales alrededor de $40 \mathrm{X} 40 \mathrm{~cm}$ como máximo para la colocación de la estructura del prototipo, esto a fin de permitir la portabilidad, este esquema también requiere una capacidad de escalabilidad en forma modular que permita la simulación de diversos procesos o la interaccion entre ellos estableciendo una línea productiva de mayor versatilidad, que además exiga por parte del usuario una 
implementación de habilidades de abstracción de la información organzacon y jerarquización de la información así como síntesis dimensional a fin de ejecutar de manera correcta los desplazamientos y operaciones a establecer por el prototipo.

Ya habiendo establecido el esquema de construcción de partida basado en maquinado por taladrado y soldadura por punto, procedemos a establecer las aplicaciones que se utilizaron como herramientas de modelado.

\subsection{Aplicación de herramientas CAE y CAD.}

A fin de establecer un prototipo funcional con respecto a demanda dinámica y cinematica para las aplicaciones respectivas, se preestablecen simulaciones de ejecución para los sistemas proyectados, para esta actividad se utilizaron las siguientes aplicaciones para la emulación del entorno de trabajo, así como parametros específicos en cuanto a rigidez, resistencia y ligereza estructural estableciendo un dispositivo robusto pero de fácil portabilidad, entre las herramientas aplicadas contamos con: G. Sketchup que nos permite bocetar en forma rápida e intuitiva los elementos de estrucutura, asi como la manipulaion de las CAD para los autómatas, FluidSim y RoboDK nos permiten aplicar la ejecusion virtual de los ciclos de trabajo del sistema además de habilitar una plataforma de programación grafica para sistemas automáticos basados en lenguajes FBD y GRAFCET, para de esta manera establecer una lógica de programación multilenguaje que sirva como fundamento para los enlaces del HMI que se desarrollaron.

A través del proceso establecido que consiste en transporte de una pieza a su punto de maquinado para posteriormente su traslado para aplicación de soldadura de punto y finalmente depositarlo a línea final de proceso, se estructuró la línea de procesamiento por medio de automatas, las unidades de manipulación y trabajo se seleccionaron atendiendo el estándar de la industria de manufactura a nivel de automatización industrial, donde se aplican los robots manipuladores, en nuestro caso un robot cartesiano por accionamiento neumático que nos pemrite desplazar las cargas con base a los tres ejes dmensionales del espacio de trabajo y un robot de brazo articulado con movimiento en un plano y rotación del mismo con base al marco de referencia de origen, estos elementos son lo considerados para la implementacion inicial.

\subsection{Selección de material estructurales.}

Teniendo los sistemas de trabajo nos enfocamos a definir los elementos que servirán de soporte estructural y sujeción de las cadenas cinematicas consideradas, aplicando para esta tarea elementos de aluminio anodizado, que permiten combinar una estructura de alta rigidez con bajo peso especifico, lo que asegura la estabilidad estructural del modelo asi como la capacidad de portabilidad y modularidad. Las características del material seleccionado permite considerar la capacidad a la fluencia del material muy por encima de las exigencias de trabajo del sistema, considerando los valores pico de presión manomética 
de la unidad de potencia la cual nos entrega una presion de 150 Psi equivalentes a 1.034 $\mathrm{MPa}$, que es la capacidad nominal del compresor que se impelenta como unidad de potencia. Con base en estos parametros de partida evaluaremos los esfuerzos cortantes como viga en dos modalidades cantiliver y doblemente apoyada. En el cual el primero es un autómata cartesiano que funge como manipulador/tranporte, asi como las estruturas de soporte /sujeción esquematizados en la Fig. 1.

(a)

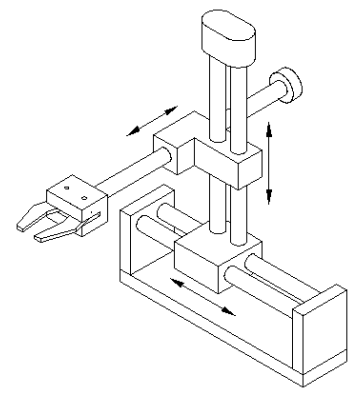

(b)

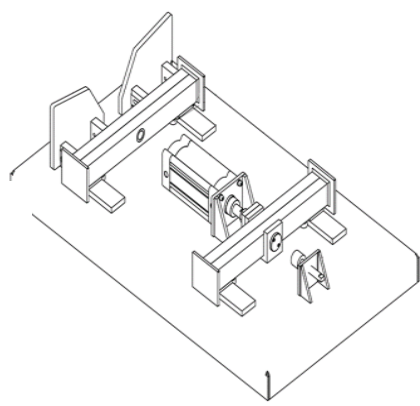

Fig. 1 (a) Estructura del autómata cartesiano (b) Sistemas de sujeción para maquinado.

Basados en este análisis, estructuramos un modelo de sistema con base en el proceso determinado, replicando el análisis previo con un modelado del sistema de orden superior utilizando la herramienta de calculo GeoGebra, de esta forma logramos establecer los graficos de desempeño del modelo estrucutural en forma virtual.

Posteriormente aplicando el modelo en la aplicación MDSolids obtenemos los valores de reacciones, cortante y flexion máximo, para de esta manera aplicar la interpretación a fin de establecer parametros de resistencia mecánica. Una vez conocidos los valores de carga se establecen valores de propiedades de los materiales seleccionados, para determinar factores de seguridad del diseño estableciéndolas con referencia a modelos que prevenga la fluencia como falla en este caso utilizamos el criterio de Von Misses.

Asi tomando los datos de resistencia mecánica del modelo propuesto obtenemos los Renders de los esfuerzos normales, como se muestra en la Fig. 2

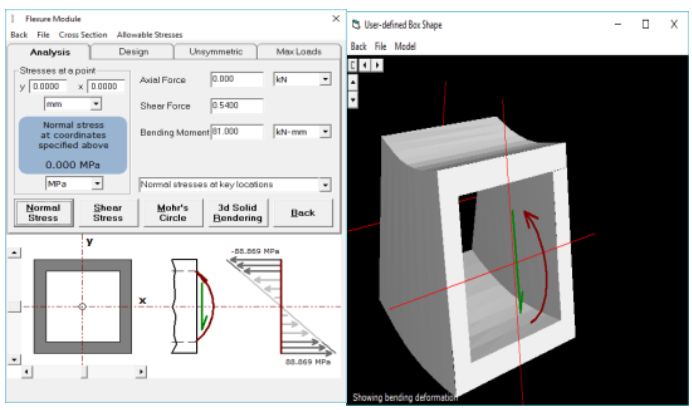

Fig. 2 (a) Render y especificación de esfuerzos normales. 
Tras los análisis implementados al diseño propuesto se hace evidente la holgura que tenemos con respecto a los valores máximos de carga que se pueden obtener, lo que nos asegura un índice de confianza por encima del $50 \%$ de fuerza para fluencia del material.

\subsection{Selección de actuadores electroneumáticos y autómatas roboticos.}

Considerando los requerimeintos de traslación y rotación del conjunto cinematico, se propone un modelo de tipo antropomórfico el cual, con cuatro grados de libertad nos permite una simplificación respecto al desplazamiento angular al mantener coplanares a dos de los eslabones de la cadena cinemática, respetando con ello el desempeño deseado, las dimensiones y flexibilidad de operación. En este caso se empleó un sistema de 4 grados de libertad a fin de ejecutar la manipulación de los elementos que emularan las materias primas del proceso dado el requerimiento de una implementación rápida se opta en primera etapa de desarrollo un diseño "open source" denominado EEEZY Bot Arm MK2 ${ }^{1}$, El cual fue modificado a fin de que los centros de gravedad y dimensiones funcionales se ajustaran a nuestro modelo de proceso.

Ahora los sistemas de potencia a utilizar en los manipuladores robóticos son los siguientes, esto considerando sus capacidades operativas así como su implementación de control: servomotor, potencia del actuador proporcional a las cargas mecánicas, la tensión o corriente que requiere depende del tamaño del servo.

\subsection{Implementación del sistema de adquisición de datos.}

De esta forma se esquematiza en la Fig. 3, la construcción del circuito de control de los actuadores con el sistema del microcontrolador esto lo implementamos a través del software fritzing, donde podemos advertir los eslabones que estarán accionándose, en la ilustración relacionamos los pines del Atmel 328 con respecto a los servos.

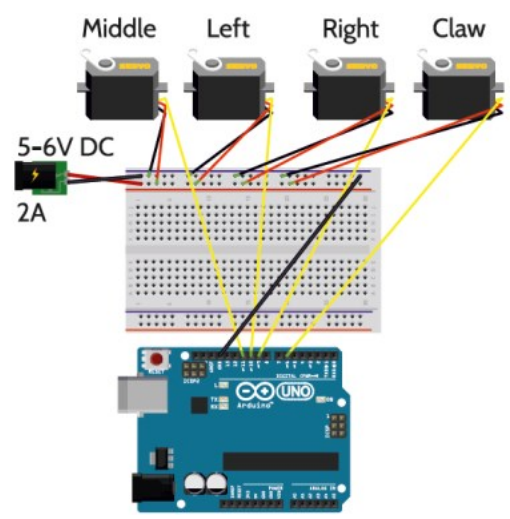

Fig. 3 Implementación del circuito Atmel 328 con servomotores

\footnotetext{
${ }^{1}$ Makerbots.thingiverse (url: https://www.thingiverse.com/thing:1454048)
} 
Como parte final del ensamblaje debemos posicionar los servos a fin de asegurar que los actuadores se configuren a los puntos de partida al ejecutar el código de accionamiento del robot.

\subsubsection{Posicionamiento de servomotores.}

Una vez montados en la estructura se procede a marcar en el cuerpo del servo una línea de referencia a fin de indicar los $90^{\circ}$ físicos del actuador, para posteriormente montar los adaptadores a posiciones de $0^{0}$ los eslabones medio y central del robot, a $90^{0}$ la tenaza y eslabón izquierdo

Antes de implementar la composición general y rutinas de trabajo del prototipo debemos establecer parámetros funcionales de cinemática directa en función de la posición deseada versus la geometría del manipulador, para esta tarea emplearemos 2 herramientas de software CAE open source, denominadas FreeCAD y RoboDK.

De esta forma de procede a resolver el PCD del robot articulado, para esto nos valemos de la aplicacion FreeCAD e implementando como sistema embebido estrucutras script en Phyton y Pyooml.Primeramente se establecen las sentencias que nos generan los marcos de referencia del sistema principal fijo y del sistema auxiliar estableciendo los vectores de posición de los compnentes del mecanismo, primero dimensionalmente para de esta manera generar las operaciones de transformación homogénea para matrices, aplicando la librería HMatrixcomo. El código se reitera para ajustar el modelo cinemático ya que el algoritmo requiere que se formulen cuatro tranformaciones homogéneas aplicables a través de dos traslaciones y dos rotaciones, a fin de construir la cadena cinematica del robot.

Una vez definido las tranformaciones respectivas los productos matriciales resultantes nos arrojan en el espacio de trabajo, la solucion en forma grafica del PCD, del autómata propuesto, estableciendo a cada posible angulos de orientación de eslabones como dato de entrada. Definidos los parametros Denavit-Hartengberg para el autómata dentro de la misma aplicación, el sistema nos permite modelar como elemento solido además de establecer el diseño CAD del eslabonamiento. Fig. 4.

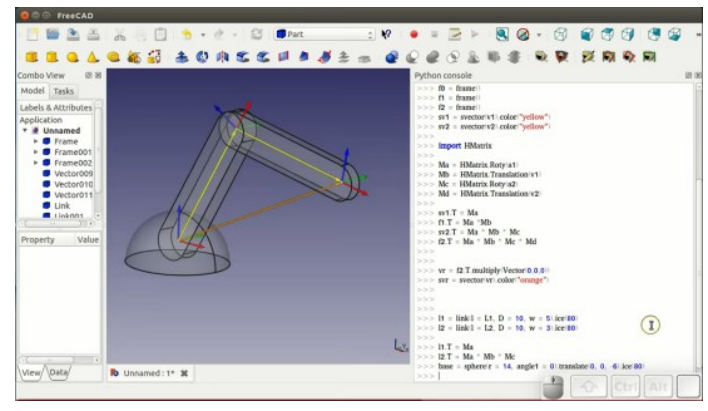

Fig. 4 Modelado CAD del solido superpuesto a los parametos Denavit-Hartenberg. 


\subsection{Programacion de autómata robotico en modalidad de "training" o "seguimiento".}

El sistema propuesto se integrara a través de algoritmos de cinematica inversa que buscan establecer los parámetros de desplazamiento angular que o permita establecer la trayectoria correcta y estable para la ejecución de las tareas del autómata en un rango confiable de repetibilidad. Para esto se utilizó el método geométrico, con el se determinaron las relaciones matemáticas para generar los angulos de entrada al autómata para la posicon del eyector deseada.

Ahora con esta parametrización estamos en condiciones de establecer el código para la modalidad training del sistema. En esta implementación aplicamos dos paradigmas de programación, en primera instancia aplicaremos el IDE del ATMEL 328, estableciendo la adquisición de datos a través de potenciómetros acondicionados como encoders de posición estableciéndolos en arreglos unidimensionales para escitura y lectura de las conversiones analogo digital. Se ejecutan estas rutinas en el automata donde se advierte un comportamiento estable del robot permitiendo su "programación de forma externa" al ordenador haciendo uso de un sistema de seguimiento emulando un aprendizaje por imitación del prototipo. Fig. 5.

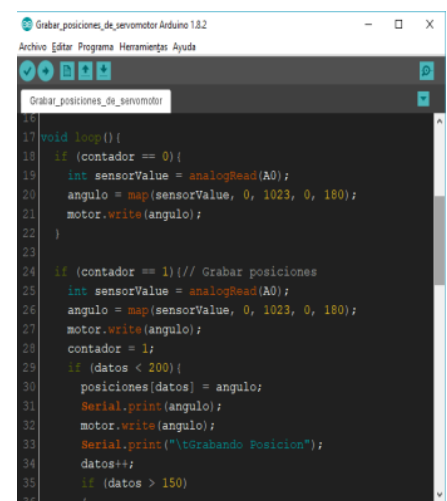

(a)

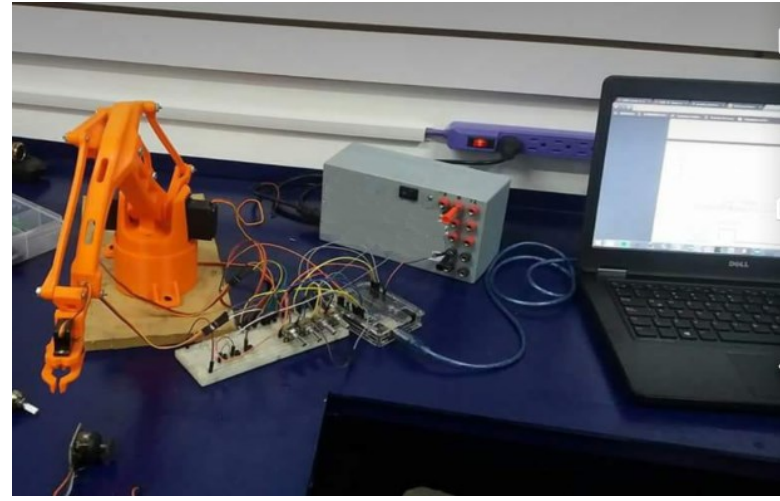

(b)

Fig. 5 a) Programacion con IDE ATMEL 328 (b) Programación externa del prototipo

Para el calculo y selección de actuadores neumáticos iniciamos definiendo el tipo de actuador seleccionado, utilizando para el presente trabajo cilindros de doble efecto.

\subsection{Implementacion de interface hombre maquina (HMI)}

Para la implementación de la inteface de contro, se utilizo paradigma visual de forma intuitiva, utilizando software open source denominada MyOpenLab utilizando la libreria Firmata. La presentación de los resultados y/o el control de las simulaciones se hace mediante un conjunto de bloques de función de visualización y/o interacción capaz de 
manejar todo tipo de datos (analógicos, digitales, matrices, vectores, imágenes, sonidos, etc.). Mediante MyOpenLab es posible diseñar instrumentos virtuales (VI) a través de los cuales se puede realizar una aproximación a los sistemas de medida y control de una manera mas realista. La realización de una simulación se hace mediante dos pantallas o áreas de trabajo: Panel Circuito y Panel Visualización.

\subsection{Ejecución de pruebas piloto y puesta a punto de prototipo.}

Para la ejecución y puesta en marcha, se enlazan los elementos del panel de control como diagrama de bloques y tras configurar los parámetros funcionales de la interfaz se puede implementar el control del autómata en tiempo real. Fig. 6

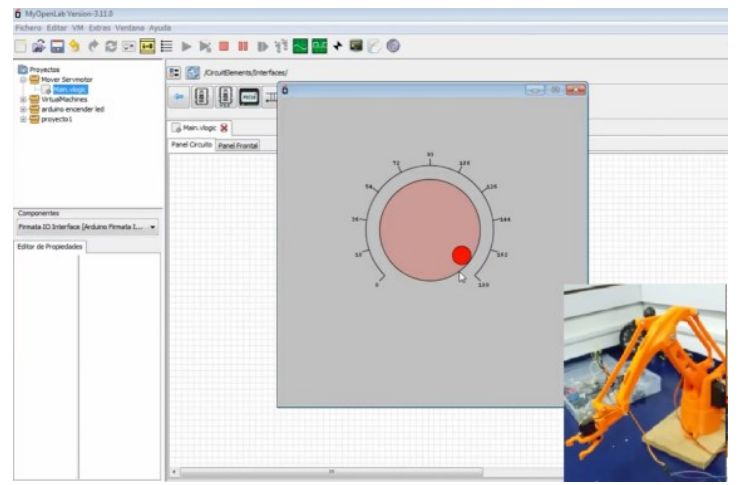

Fig. 6 Pantallas de inteface de control y comunicación con automata fisico.

\section{Resultados Finales}

Finalmente se muestran los resultados obtenidos, ejecutando el prototipo robotico para el proceso de manufactura propuesto, se propone para trabajos futuros la implementación de otros procesos de manufactura que pueden ser añadidos, tales como pintado y/o grabado de placas y la implementación de técnicas de inteligencia artificial que permita el reconocimiento de patrones para identificar limites o formas de las estructuras utilizadas. El prototipoo integrado se muestra en la Fig. 7.

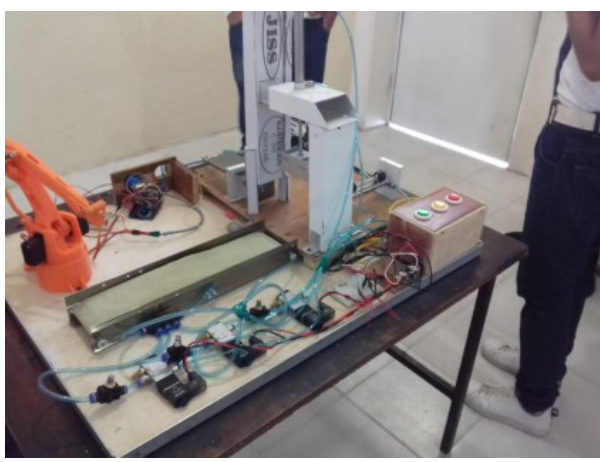

Fig. 7 Presentación final del prototipo de celula robotica para proceso de manufactura 


\section{Referencias}

Assembly Manual for V1.0 MeArm.io, revisión 1.4, doi: http://learn.mime.co.uk/assets/docs/buildingthe-mearm-deluxe/MeArm_v1.0_Manual_v1.4.pdf. Septiembre 2015.

D. Alimisis (2009). Teacher Education on Robotics-Enhanced Constructivist Pedagogical Methods. School of Pedagogical and Technological Education (ASPETE).. ISBN 978-960-6749-49-0.2009. [Online]. Available: http://dide.ilei.sch.gr/keplinet/education/docs/ book_TeacherEducationOnRobotics-ASPETE.pdf

E. Montiel, G. Cordova, F. Paredes, P. Campos, P. "Diseño y Construcción de un Prototipo de Robot Educativo para Apoyo de la Enseñanza de los Números a Nivel Preescolar”. La Mecatrónica en México, vol. 5, No. 2, páginas 47 - 55. Mayo 2016.

F. Alonzo, "Diseño, construcción y control de un brazo robótico", Ingenieria en electrónica. Colegio de Ciencias e Ingenieria, Universidad San Francisco de Quito. Diciembre de 2014.

K. Waldron, J. Schmiedeler, J(2008). "Springer Handbook of Robotics": 9-33. ISBN 978-3-54023957-4. [Online]. Available: http://www.wikiwand.com/pt/Par\%C3\%A2metros_de_DenavitHartenberg

M. Spong, M. Vidyasagar(1989 January). Robot Dynamics and Control. New York: John Wiley \& Sons. ISBN 978-0-471-61243-8.

Parra C, Bravo F, García L (2013), "Generación de Ambientes de Aprendizaje Interdisciplinarios con Robótica en Instituciones Educativas de Bajos Recursos Económicos”, Presentado en WEEF, Cartagena, Colombia.[Online].Available: https://www.acofipapers.org/index.php/ acofipapers/2013/paper/viewFile/219/116.

P. Richard (1981, Ene 1). Robot manipulators: mathematics, programming, and control : the computer control of robot manipulators. Cambridge, MA: MIT Press. ISBN 97 8-0-262-16082-7.

[Online]. Available: https:// www.amazon.es/Robot-Manipulators-Mathematics-ProgrammingIntelligence/dp/026216082X.

W. Khalil, E. Dombre (2004, July 1). Modeling, identification and control of robots. New York: Taylor Francis. ISBN 1-56032-983-1. 